\title{
Prevention and Treatment of Opportunistic Infection
}

National Cancer Institute

\section{Source}

National Cancer Institute. Prevention and Treatment of Opportunistic Infection. NCI

Thesaurus. Code 15840.

Improve understanding of the biology of opportunistic pathogens, identify potential molecular targets for effective therapies, and discover and delineate the structure and function of potential agents for prevention and treatment of HIV-associated Ols and other infections that are modified by HIV infection. 